Pacific Journal of Mathematics

ABEL-ERGODIC THEOREMS FOR SUBSEQUENCES 


\section{ABEL-ERGODIC THEOREMS FOR SUBSEQUENCES}

\section{RYotaRo SATO}

\section{Let $T$ be a positive linear contraction on an $L^{1}$-space and} $k_{1}, k_{2}, \cdots$ an increasing sequence of positive integers. In this paper the almost everywhere convergence of Abel averages $\sum_{i=1}^{\infty} r^{k_{i}} T^{k_{i}} f / \sum_{i=1}^{\infty} r^{k_{i}}$ for the sequence $k_{1}, k_{2}, \cdots$ as $r \uparrow 1$ is investigated.

In [3], A. Brunel and M. Keane defined uniform sequences for increasing sequences of positive integers and proved that if $\phi$ is a measure preserving transformation on a finite measure space then for any uniform sequence $k_{1}, k_{2}, \ldots$ and for any integrable function $f$, Cesàro averages of $f\left(\phi^{k i} \cdot\right)$ converge almost everywhere. The author [13], [14] has recently generalized and extended this result to one at the operator theoretic level. On the other hand, the work of G.-C. Rota [11] suggests that it would be of interest to consider the almost everywhere convergence of Abel averages for uniform sequences. These are the starting points for the study in this paper.

2. Main results. Let $(\Omega, \mathscr{B}, m)$ be a $\sigma$-finite measure space with positive measure $m$ and $L^{p}(\Omega)=L^{p}(\Omega, \mathscr{B}, m), 1 \leqq p \leqq \infty$, the usual (complex) Banach spaces. Let $T$ be a positive linear operator from $L^{1}(\Omega)$ to $L^{1}(\Omega)$ with $\|T\|_{1} \leqq 1$. We shall say that the Abelindividual ergodic theorem holds for $T$ if for any uniform sequence $k_{1}, k_{2}, \cdots$ (for the definition, see [3]) and for any $f \in L^{1}(\Omega)$, the limit

$$
\widetilde{f}(\omega)=\lim _{r \uparrow 1} \frac{\sum_{i=1}^{\infty} r^{k_{i}} T^{k_{i}} f(\omega)}{\sum_{i=1}^{\infty} r^{k_{i}}}
$$

exists almost everywhere and $\tilde{f} \in L^{1}(\Omega)$. The main results of this paper are the following two theorems.

THEOREM 1. If $T$ maps, in addition, $L^{p}(\Omega)$ into $L^{p}(\Omega)$ for some $p$ with $1<p<\infty$ and $\|T\|_{p} \leqq 1$, then the Abel-individual ergodic theorem holds for $T$.

THEOREM 2. If there exists a strictly positive function $h \in L^{1}(\Omega)$ such that the set

$$
\left\{(1-r) \sum_{k=0}^{\infty} r^{k} T^{k} h ; 0<r<1\right\}
$$

is weakly sequentially compact in $L^{1}(\Omega)$, then the Abel-individual ergodic theorem holds for $T$. 
In $\S 4$ it is proved that if $T$ maps, in addition, $L^{\infty}(\Omega)$ into $L^{\infty}(\Omega)$ and $\|T\|_{\infty} \leqq 1$, then the Abel-maximal ergodic theorem holds for $T$; i.e., for any uniform sequence $k_{1}, k_{2}, \cdots$ and for any $f \in L^{p}(\Omega)$ with $1<p<\infty$, the function $f^{*}$ defined by

$$
f^{*}(\omega)=\sup _{0<r<1}\left|\frac{\sum_{i=1}^{\infty} r^{k_{i}} T^{k_{i}} f(\omega)}{\sum_{i=1}^{\infty} r^{k_{i}}}\right|
$$

belongs of $L^{p}(\Omega)$. The last section is concerned with point transformations from $\Omega$ into $\Omega$. A necessary and sufficient condition that a measure preserving transformation on a probability space be weakly mixing is given in terms of Abel-ergodic limits.

\section{Proofs of the main theorems.}

3.1. Proof of Theorem 1. Our proof is similar to that given in [14]. Let $k_{1}, k_{2}, \cdots$ be a uniform sequence, and let $(X, \mathscr{X}, \mu, \varphi)$ and $y, Y$ be the apparatus [3] connected with this sequence. $\Phi$ will denote the operator on $L^{1}(X)$ induced by $\varphi$. Taking $\left(\Omega^{\prime}, \mathscr{B}^{\prime}, m^{\prime}\right)$ to be the direct product of $(\Omega, \mathscr{B}, m)$ and $(X, \mathscr{X}, \mu)$ and $T^{\prime}$ the direct product of $T$ and $\Phi$, it follows that $T^{\prime}$ is a positive linear operator from $L^{1}\left(\Omega^{\prime}\right)$ to $L^{1}\left(\Omega^{\prime}\right)$ and $\left\|T^{\prime}\right\|_{1} \leqq 1$. Since $\|T\|_{p} \leqq 1$ by hypothesis, it also follows that $T^{\prime}$ maps $L^{p}\left(\Omega^{\prime}\right)$ into $L^{p}\left(\Omega^{\prime}\right)$ and $\left\|T^{\prime}\right\|_{p} \leqq 1$.

Suppose first that $f \in L^{1}(\Omega) \cap L^{p}(\Omega)$ and $f \geqq 0$. As in [3], for any fixed $\varepsilon>0$, choose open subsets $Y^{\prime}, Y^{\prime \prime}$ and $W$ of $X$ such that $Y^{\prime} \subset Y \subset Y^{\prime \prime}, \mu\left(Y^{\prime \prime}-Y^{\prime}\right)<\varepsilon, y \in W$, and for any $x \in W$ and any $n \geqq 0$,

$$
1_{Y^{\prime}}\left(\varphi^{n} x\right) \leqq 1_{Y}\left(\varphi^{n} y\right) \leqq 1_{Y^{\prime \prime}}\left(\varphi^{n} x\right)
$$

Define

$$
\begin{aligned}
g(\omega, x) & =f(\omega) 1_{Y}(x), \\
g^{\prime}(\omega, x) & =f(\omega) 1_{Y^{\prime}}(x)
\end{aligned}
$$

and

$$
g^{\prime \prime}(\omega, x)=f(\omega) 1_{Y^{\prime \prime}}(x) .
$$

Since every Cesàro summable sequence is Abel summable (see, for example, [16, Chapter III]), it follows from [1] that

$$
\widetilde{g}^{\prime}(\omega, x)=\lim _{r \uparrow 1}(1-r) \sum_{k=0}^{\infty} r^{k} T^{\prime k} g^{\prime}(\omega, x)
$$

and

$$
\widetilde{g}^{\prime \prime}(\omega, x)=\lim _{r \uparrow 1}(1-r) \sum_{k=0}^{\infty} r^{k} T^{\prime k} g^{\prime \prime}(\omega, x)
$$


exist and are finite almost everywhere. Clearly $\widetilde{g}^{\prime}$ and $\widetilde{g}^{\prime \prime}$ belong to $L^{p}\left(\Omega^{\prime}\right)$. It also follows from Cohen's mean ergodic theorem [7] that

$$
\lim _{r \uparrow 1}\left\|(1-r) \sum_{k=0}^{\infty} r^{k} T^{\prime k} g^{\prime}-\widetilde{g}^{\prime}\right\|_{p}=0
$$

and

$$
\lim _{r \uparrow 1}\left\|(1-r) \sum_{k=0}^{\infty} r^{k} T^{\prime k} g^{\prime \prime}-\widetilde{g}^{\prime \prime}\right\|_{p}=0
$$

Put

$$
S(\omega)=\limsup _{r \uparrow 1}(1-r) \sum_{k=0}^{\infty} r^{k} T^{k} f(\omega) 1_{Y}\left(\varphi^{k} y\right)
$$

and

$$
s(\omega)=\liminf _{r \uparrow 1}(1-r) \sum_{k=0}^{\infty} r^{k} T^{k} f(\omega) 1_{Y}\left(\varphi^{k} y\right) .
$$

Since $T$ is positive, it follows that

$$
\widetilde{g}^{\prime}(\omega, x) \leqq s(\omega) \leqq S(\omega) \leqq \widetilde{g}^{\prime \prime}(\omega, x)
$$

almost everywhere on $\Omega \times W$. Thus for any $\Omega_{1} \in \mathscr{B}$ with $m\left(\Omega_{1}\right)<\infty$ we have

$$
\begin{aligned}
\int_{\Omega_{1}} & (S(\omega)-s(\omega)) d m(\omega) \\
& =\mu(W)^{-1} \int_{\Omega_{1} \times W}(S(\omega)-s(\omega)) d m^{\prime}(\omega, x) \\
& \leqq \mu(W)^{-1} \int_{\Omega_{1} \times W}\left(\widetilde{g}^{\prime \prime}-\widetilde{g}^{\prime}\right) d m^{\prime} \\
& =\mu(W)^{-1} \lim _{r \uparrow 1} \int_{\Omega_{1} \times W}(1-r) \sum_{k=0}^{\infty} r^{k} T^{k} f(\omega) 1_{Y^{\prime \prime}-Y^{\prime}}\left(\varphi^{k} x\right) d m^{\prime}(\omega, x) \\
& \leqq \mu(W)^{-1}\|f\|_{1} \int_{W} \lim _{r \uparrow 1}(1-r) \sum_{k=0}^{\infty} r^{k} 1_{Y^{\prime \prime}-Y^{\prime}}\left(\varphi^{k} x\right) d \mu(x) \\
& =\varepsilon\|f\|_{1} .
\end{aligned}
$$

Since $\varepsilon>0$ is arbitrary, this demonstrates that $S(\omega)=s(\omega)$ almost everywhere on $\Omega_{1}$. Since $(\Omega, \mathscr{B}, m)$ is a $\sigma$-finite measure space, we conclude that

$$
\begin{aligned}
\widetilde{S}(\omega) & =\lim _{r \uparrow 1}(1-r) \sum_{k=0}^{\infty} r^{k} T^{k} f(\omega) 1_{Y}\left(\Phi^{k} y\right) \\
& =\lim _{r \uparrow 1}(1-r) \sum_{i=1}^{\infty} r^{k_{i}} T^{k i} f(\omega)
\end{aligned}
$$

exists and is finite almost everywhere. On the other hand, it is known [3] that 


$$
\lim _{i \rightarrow \infty} \frac{i}{k_{i}}=\mu(Y)
$$

from which it follows that

$$
\lim _{r \uparrow 1}(1-r) \sum_{i=1}^{\infty} r^{k_{i}}=\mu(Y) \text {. }
$$

Therefore,

$$
\begin{aligned}
\tilde{f}(\omega) & =\lim _{r \uparrow 1} \frac{\sum_{i=1}^{\infty} r^{k_{i}} T^{k_{i}} f(\omega)}{\sum_{i=1}^{\infty} r^{k_{i}}} \\
& =\lim _{r \uparrow 1} \frac{(1-r) \sum_{i=1}^{\infty} r^{k_{i}} T^{k_{i}} f(\omega)}{(1-r) \sum_{i=1}^{\infty} r^{k_{i}}}
\end{aligned}
$$

exists and is finite almost everywhere.

Next suppose that $f \in L^{1}(\Omega)$. It can be easily seen that

$$
\sup _{0<r<1}\left|\frac{\sum_{i=1}^{\infty} r^{k_{i}} T^{k_{i}} f(\omega)}{\sum_{i=1}^{\infty} r^{k_{i}}}\right|<\infty
$$

almost everywhere. Since $L^{1}(\Omega) \cap L^{p}(\Omega)$ is dense in $L^{1}(\Omega)$ in the norm topology and for almost every $\omega \in \Omega$ the series $\sum_{i=1}^{\infty} r^{k_{i}} T^{k_{i}} f(\omega)$ has at least unit radius of convergence as a power series in $r$, it follows from the Banach convergence theorem [8] that for any $f \in L^{1}(\Omega)$, the limit

$$
\widetilde{f}(\omega)=\lim _{r \uparrow 1} \frac{\sum_{i=1}^{\infty} r^{k_{i}} T^{k_{i}} f(\omega)}{\sum_{i=1}^{\infty} r^{k_{i}}}
$$

exists and is finite almost everywhere. Fatou's lemma implies now that $\tilde{f} \in L^{1}(\Omega)$. This completes the proof of Theorem 1 .

3.2. Proof of Theorem 2. If we define an integrable function $h^{\prime}$ on $\Omega^{\prime}=\Omega \times X$ by $h^{\prime}(\omega, x)=h(\omega)$, then the set

$$
\left\{(1-r) \sum_{k=0}^{\infty} r^{k} T^{\prime k} h^{\prime} ; 0<r<1\right\}
$$

is weakly sequentially compact in $L^{1}\left(\Omega^{\prime}\right)$. Thus Cohen's mean ergodic theorem [7] implies that there exists a function $g^{\prime}$ in $L^{1}\left(\Omega^{\prime}\right)$ such that $T^{\prime} g^{\prime}=g^{\prime}$ and

$$
\lim _{r \uparrow 1}\left\|(1-r) \sum_{k=0}^{\infty} r^{k} T^{\prime k} h^{\prime}-g^{\prime}\right\|_{1}=0 \text {. }
$$

Clearly $g^{\prime} \geqq 0$. Let us denote $A^{\prime}=\left\{(\omega, x) \in \Omega^{\prime} ; g^{\prime}(\omega, x)=0\right\}$. We shall first prove that $A^{\prime}$ coincides with the dissipative part [5] of $T^{\prime}$. In fact, since $g^{\prime}$ is invariant under $T^{\prime}$, it follows at once that $T^{\prime *} 1_{A^{\prime}} \leqq$ 
$1_{A^{\prime}}$ where $T^{*}$ denotes the corresponding adjoint operator on $L^{1}\left(\Omega^{\prime}\right)^{*}=$ $L^{\infty}\left(\Omega^{\prime}\right)$. Hence if we define $B^{\prime}=A^{\prime} \cap C^{\prime}$, where $C^{\prime}$ denotes the conservative part [5] of $T^{\prime}$, then $T^{* *} 1_{B^{\prime}}=1_{B^{\prime}}$ on $C^{\prime}$. Thus

$$
\begin{aligned}
\int h^{\prime} 1_{B^{\prime}} d m^{\prime} & \leqq \lim _{r \uparrow 1} \int(1-r) \sum_{k=0}^{\infty} r^{k} h^{\prime}\left(T^{*}\right)^{k} 1_{B^{\prime}} d m^{\prime} \\
& =\lim _{r \uparrow 1} \int\left[(1-r) \sum_{k=0}^{\infty} r^{k} T^{\prime k} h^{\prime}\right] 1_{B^{\prime}} d m^{\prime} \\
& =\int g^{\prime} 1_{B^{\prime}} d m^{\prime}=0 .
\end{aligned}
$$

Since $h^{\prime}$ is strictly positive, it follows that $m^{\prime}\left(B^{\prime}\right)=0$. Consequently $A^{\prime} \subset \Omega^{\prime}-C^{\prime}$. On the other hand, it is clear that $A^{\prime} \supset Q^{\prime}-C^{\prime}$.

Let $f^{\prime}$ be any function in $L^{1}\left(\Omega^{\prime}\right)$. It follows that

$$
\tilde{f}^{\prime}(\omega, x)=\lim _{r \uparrow 1}(1-r) \sum_{k=0}^{\infty} r^{k} T^{\prime k} f^{\prime}(\omega, x)=0
$$

almost everywhere on $A^{\prime}$. On the other hand,

$$
\begin{aligned}
\tilde{f}^{\prime}(\omega, x) & =\lim _{r \uparrow 1}(1-r) \sum_{k=0}^{\infty} r^{k} T^{\prime k} f^{\prime}(\omega, x) \\
& \left.=g^{\prime} \omega, x\right) \lim _{r \uparrow 1} \frac{\sum_{k=0}^{\infty} r^{k} T^{\prime k} f^{\prime}(\omega, x)}{\sum_{k=0}^{\infty} r^{k} T^{\prime k} g^{\prime}(\omega, x)}
\end{aligned}
$$

exists and is finite almost everywhere on $\Omega^{\prime}-A^{\prime}$, since the right hand side of the last formula exists and is finite almost everywhere on $\Omega^{\prime}-A^{\prime}$ by the ergodic theorem of Báez-Duarte [2]. This together with the fact that the average

$$
(1-r) \sum_{k=0}^{\infty} r^{k} T^{\prime k} f^{\prime}
$$

converges in the norm of $L^{1}\left(\Omega^{\prime}\right)$ to a function in $L^{1}\left(\Omega^{\prime}\right)$ as $r \uparrow 1$, which may be proved by a slight modification of an argument in [10], implies that

$$
\lim _{r \uparrow 1}\left\|(1-r) \sum_{k=0}^{\infty} r^{k} T^{\prime k} f^{\prime}-\widetilde{f}^{\prime}\right\|_{1}=0 .
$$

Therefore, an argument analogous to that in the proof of Theorem 1 is sufficient to prove the present theorem, and we omit the details.

4. The Abel-maximal ergodic theorem. Throughout this section it is assumed that $T$ maps, in addition, $L^{\infty}(\Omega)$ into $L^{\infty}(\Omega)$ and $\|T\|_{\infty} \leqq 1$. It follows from the Riesz convexity theorem that $T$ maps $L^{p}(\Omega)$ into $L^{p}(\Omega)$ for each $p$ with $1 \leqq p \leqq \infty$ and $\|T\|_{p} \leqq 1$. Let $f$ be a function in $L^{p}(\Omega)$ and $a>0$. Following R. V. Chacon [4], we define 


$$
\begin{aligned}
& f^{a-}(\omega)=[\operatorname{sgn} f(\omega)] \min (a,|f(\omega)|), \\
& f^{a+}(\omega)=[\operatorname{sgn} f(\omega)](|f(\omega)|-\min (a,|f(\omega)|))
\end{aligned}
$$

and

$$
E^{*}(a)=\left\{\omega ; \sup _{0<r<1}\left|(1-r) \sum_{k=0}^{\infty} r^{k} T^{k} f(\omega)\right|>a\right\},
$$

where $\operatorname{sgn} f(\omega)=f(\omega) /|f(\omega)|$ if $f(\omega) \neq 0$ and $\operatorname{sgn} f(\omega)=0$ if $f(\omega)=0$.

The following lemma is the Abel-analogue of Chacon's maximal ergodic lemma [4].

Lemma. If $1 \leqq p<\infty$ and $f \in L^{p}(\Omega)$ then for each $a>0$ we have

$$
\int_{E^{*}(a)}\left(a-\left|f^{a-}(\omega)\right|\right) d m(\omega) \leqq \int\left|f^{a+}(\omega)\right| d m(\omega) .
$$

Proof. It may and will be assumed without loss of generality that $f$ is a nonnegative function. Let $\omega \in E^{*}(a)$. Then it follows that $\sup _{0<r<1} \sum_{k=0}^{\infty} r^{k}\left(T^{k} f(\omega)-a\right)>0$. Hence there exists a positive real $r$ with $r<1$ and an integer $n \geqq 0$ such that

$$
\sum_{k=0}^{n} r^{k}\left(T^{k} f(\omega)-a\right)>0
$$

and

$$
\sum_{k=0}^{j} r^{k}\left(T^{k} f(\omega)-a\right) \leqq 0 \text { for } 0 \leqq j<n .
$$

But this implies [2] that

$$
\frac{1}{n+1} \sum_{k=0}^{n} T^{k} f(\omega)>a .
$$

Hence Chacon's maximal ergodic lemma [4] completes the proof of the present lemma.

THEOREM 3. If $1<p<\infty, f \in L^{p}(\Omega)$ and $k_{1}, k_{2}, \cdots$ is a uniform sequence, then the function $f^{*}$ defined by

$$
f^{*}(\omega)=\sup _{0<r<1}\left|\frac{\sum_{i=1}^{\infty} r^{k_{i}} T^{k_{i}} f(\omega)}{\sum_{i=1}^{\infty} r^{k_{i}}}\right|
$$

belongs to $L^{p}(\Omega)$.

Before the proof we note that the positivity of $T$ is not necessary in this theorem. This follows from [6].

Proof. It may and will be assumed without loss of generality that $f$ is a nonnegative function. Since 


$$
\lim _{r \uparrow 1}(1-r) \sum_{i=1}^{\infty} r^{k_{i}}=\mu(Y)>0,
$$

it suffices to prove that the function $h^{*}$ defined by

$$
h^{*}(\omega)=\sup _{0<r<1}(1-r) \sum_{k=0}^{\infty} r^{k} T^{k} f(\omega)
$$

belongs to $L^{p}(\Omega)$. But it follows easily from the previous lemma that for each $a>0$,

$$
m\left(\left\{h^{*}>a\right\}\right) \leqq \frac{1}{a} \int_{\left\{h^{*}>a\right\}}|f| d m<\infty .
$$

Thus Theorem 2.2.3 in [9] completes the proof of Theorem 3.

Using Theorem 1, it may be readily seen that for any uniform sequence $k_{1}, k_{2}, \cdots$ and for any $f \in L^{p}(\Omega)$ with $1 \leqq p<\infty$, the limit

$$
\widetilde{f}(\omega)=\lim _{r \uparrow 1} \frac{\sum_{i=1}^{\infty} r^{k_{i}} T^{k_{i}} f(\omega)}{\sum_{i=1}^{\infty} r^{k_{i}}}
$$

exists and is finite almost everywhere. This together with the above theorem implies at once the following Abel-mean ergodic theorem.

THEOREM 4. For any uniform sequence $k_{1}, k_{2}, \cdots$ and for any $f \in L^{p}(\Omega)$ with $1<p<\infty$, we have

$$
\lim _{r \uparrow 1}\left\|\frac{\sum_{i=1}^{\infty} r^{k_{i}} T^{k_{i}} f}{\sum_{i=1}^{\infty} r^{k_{i}}}-\tilde{f}\right\|_{p}=0
$$

\section{Applications to point transformations.}

THEOREM 5. Let $\phi$ be a point transformation from $\Omega$ into $\Omega$ such that $\phi^{-1} A \in \mathscr{B}$ if $A \in \mathscr{B}$ and $m\left(\phi^{-1} A\right)=0$ if $m(A)=0$. Suppose there exists a constant $K$ such that

$$
0<\lim \sup _{n} \frac{1}{n} \sum_{k=0}^{n-1} m\left(\phi^{-k} A\right) \leqq K m(A)
$$

for every measurable set $A$ of positive measure. Then for any uniform sequence $k_{1}, k_{2}, \cdots$ and for any $f \in L^{p}(\Omega)$ with $1 \leqq p<\infty$, the limit

$$
\widetilde{f}(\omega)=\lim _{r \uparrow 1} \frac{\sum_{i=1}^{\infty} r^{k_{i}} f\left(\phi^{k_{i}} \omega\right)}{\sum_{i=1}^{\infty} r^{k_{i}}}
$$

exists almost everywhere and $\tilde{f} \in L^{p}(\Omega)$.

Proof. It follows from [12] and [15] that there exists a $\sigma$-finite measure $\nu$ on $(\Omega, \mathscr{B})$ such that 
(a) $\nu(A) \leqq K^{2} m(A)$ for all $A \in \mathscr{B}$;

(b) $\nu(A) \geqq m(A)$ for $A \in \mathscr{B}$ with $A=\phi^{-1} A$;

(c) $\nu(A)=0$ if and only if $m(A)=0$;

(d) $\nu$ is invariant under $\phi$.

Therefore, if $f \in L^{p}(\Omega, \mathscr{B}, m)$ then, by (a), $f \in L^{p}(\Omega, \mathscr{B}, \nu)$. Since $\phi$ is $\nu$-measure preserving, it follows from the previous arguments that the limit (1) exists and is finite $\nu$-almost everywhere. This together with (c) implies the $m$-almost everywhere convergence of (1). To prove that $\tilde{f} \in L^{p}(\Omega, \mathscr{B}, m)$, it suffices to show that for any nonnegative function $f$ in $L^{p}(\Omega, \mathscr{B}, m)$, the function $\tilde{h}$ defined by

$$
\tilde{h}(\omega)=\lim _{r \uparrow 1}(1-r) \sum_{k=0}^{\infty} r^{k} f\left(\phi^{k} \omega\right)
$$

belongs to $L^{p}(\Omega, \mathscr{B}, m)$. But, clearly, $\tilde{h} \in L^{p}(\Omega, \mathscr{B}, \nu)$ is invariant under $\phi$, and hence (b) implies $\tilde{h} \in L^{p}(\Omega, \mathscr{B}, m)$. The proof is complete.

From now on it is assumed that $(\Omega, \mathscr{B}, m)$ is a probability space and $\phi$ is a measure preserving transformation on $(\Omega, \mathscr{B}, m)$. The transformation $\phi$ is called ergodic if $A \in \mathscr{B}$ and $\phi^{-1} A=A$ imply $m(A)=0$ or $m(A)=1$; weakly mixing if for each pair $A, B \in \mathscr{B}$, we have

$$
\lim _{n} \frac{1}{n} \sum_{k=0}^{n-1}\left|m\left(\phi^{-k} A \cap B\right)-m(A) m(B)\right|=0 ;
$$

strongly mixing if for each pair $A, B \in \mathscr{B}$, we have

$$
\lim _{k} m\left(\phi^{-k} A \cap B\right)=m(A) m(B) \text {. }
$$

THEOREM 6. For a measure preserving transformation on a probability space $(\Omega, \mathscr{B}, m)$, the following three statements are equivalent:

$(\alpha) \phi$ is weakly mixing.

( $\beta$ ) For any uniform sequence $k_{1}, k_{2}, \cdots$ and for any $f \in L^{1}(\Omega)$, we have

$$
\tilde{f}(\omega)=\int f d m \text { almost everywhere } .
$$

( $\gamma$ ) For any uniform sequence $k_{1}, k_{2}, \cdots$ and for any $f \in L^{1}(\Omega)$, we have

$$
\lim _{r \uparrow 1}\left\|\frac{\sum_{i=1}^{\infty} r^{k_{i}} f\left(\dot{\phi}^{k_{i}} \omega\right)}{\sum_{i=1}^{\infty} r^{k_{i}}}-\int f d m\right\|_{1}=0
$$

Proof. $(\alpha)$ implies $(\beta)$ : In the proof of Theorem 1, if we define the measure preserving transformation $\phi^{\prime}$ on $\left(\Omega^{\prime}, \mathscr{B}^{\prime}, m^{\prime}\right)$ by 


$$
\phi^{\prime}(\omega, x)=(\phi \omega, \varphi x),
$$

then $(\alpha)$ implies that $\phi^{\prime}$ is ergodic (cf. [3]). Hence for any nonnegative function $f \in L^{1}(\Omega)$ we have

$$
\lim _{r \uparrow 1}(1-r) \sum_{k=0}^{\infty} r^{k} f\left(\phi^{k} \omega\right) 1_{Y^{\prime}}\left(\varphi^{k} x\right)=\mu\left(Y^{\prime}\right) \int f d m
$$

and

$$
\lim _{r \uparrow 1}(1-r) \sum_{k=0}^{\infty} r^{k} f\left(\phi^{k} \omega\right) 1_{Y^{\prime \prime}}\left(\Phi^{k} x\right)=\mu\left(Y^{\prime \prime}\right) \int f d m
$$

almost everywhere with respect to $m^{\prime}$, from which $(\beta)$ follows immediately.

$(\beta)$ implies $(\gamma)$ : Obvious.

$(\gamma)$ implies $(\alpha)$ : Suppose that $(\gamma)$ is true but $\phi$ is not weakly mixing. Then there exists a bounded function $f$ in $L^{2}(\Omega)$ such that $\|f\|_{2}=1, \int_{f} f d m=0, f(\phi \omega)=c f(\omega)$ almost everywhere for some constant $c$ with $|c|=1$. Define a uniform sequence $k_{1}, k_{2}, \cdots$ recursively as:

$$
\begin{aligned}
& k_{1}=\min \left\{j \geqq 1 ;-\pi / 4<\arg \left(c^{j}\right)<\pi / 4\right\}, \\
& k_{n}=\min \left\{j>k_{n-1} ;-\pi / 4<\arg \left(c^{j}\right)<\pi / 4\right\} .
\end{aligned}
$$

It follows that for each positive real $r$ with $r<1$,

$$
\begin{aligned}
& \operatorname{Re} \int \frac{f(\omega) \sum_{i=1}^{\infty} r^{k_{i}} f\left(\phi^{k_{i}} \omega\right)}{\sum_{i=1}^{\infty} r^{k_{i}}} d m(\omega)=\operatorname{Re}\left(\frac{\sum_{i=1}^{\infty} r^{k_{i}} c^{k_{i}}}{\sum_{i=1}^{\infty} r^{k_{i}}}\right) \\
& \quad \geqq \frac{1}{\sqrt{2}} .
\end{aligned}
$$

Since $f$ is bounded, this contradicts our assumption, and hence $\phi$ must be weakly mixing. This completes the proof of Theorem 6 .

\section{REFERENCES}

1. M. A. Akcoglu and R. V. Chacon, A convexity theorem for positive operators, Z. Wahrscheinlichkeitstheorie verw. Geb., 3 (1965), 328-332.

2. L. Báez-Duarte, An ergodic theorem of Abelian type, J. Math. Mech., 15 (1966), 599-607.

3. A. Brunel and M. Keane, Ergodic theorems for operator sequences, Z. Wahrscheinlichkeitstheorie verw. Geb., 12 (1969), 231-240.

4. R. V. Chacon, An ergodic theorem for operators satisfying norm conditions, J. Math. Mech., 11 (1962), 165-172.

$5 . \quad$ Identification of the limit of operator averages, J. Math. Mech., 11 (1962), 961-968.

6. R. V. Chacon and U. Krengel, Linear modulus of a linear operator, Proc. Amer. Math. Soc., 15 (1964), 553-559.

7. L. W. Cohen, On the mean ergodic theorem, Ann. of Math., (2) 41 (1940), 505-509. 
8. N. Dunford and J. T. Schwartz, Convergence almost everywhere of operator averages, J. Rat. Mech. Anal., 5 (1956), 129-178.

9. A. M. Garsia, Topics in Almost Everywhere Convergence, Markham Publishing Co., Chicago, 1970.

10. Y. Ito, Uniform integrability and the pointwise ergodic theorem, Proc. Amer. Math. Soc., 16 (1965), 222-227.

11. G.-C. Rota, On the maximal ergodic theorem for Abel-limits, Proc. Amer. Math. Soc., 14 (1963), 722-723.

12. C. Ryll-Nardzewski, On the ergodic theorems. I. (Generalized ergodic theorems), Studia Math., 12 (1951), 65-73.

13. R. Sato, On a decomposition of transformations in infinite measure spaces, Pacific J. Math., 44 (1973), 733-738.

14. - On the individual ergodic theorem for subsequences, Studia Math., 45 (1973), 31-35.

15. S. Tsurumi, On the ergodic theorem, Tôhoku Math. J., (2) 6 (1954), 53-68.

16. A. Zygmund, Trigonometric Series, Vol, I, Cambridge University Press, Cambridge, 1959.

Received March 10, 1972.

Josai University, SAKado, Saitama 350-02, Japan 


\section{PACIFIC JOURNAL OF MATHEMATICS}

\section{EDITORS}

D. Gilbarg and J. Milgram

Stanford University

Stanford, California 94305

R. A. Beaumont

University of Washington

Seattle, Washington 98105
J. DUGUNDJI* Department of Mathematics

University of Southern California Los Angeles, California 90007

RICHARD ARENS

University of California

Los Angeles, California 90024

\section{ASSOCIATE EDITORS}

E. F. BeCKenbach

B. H. NeUMaNN

F. WOLF

K. Yoshida

\section{SUPPORTING INSTITUTIONS}

UNIVERSITY OF BRITISH COLUMBIA

UNIVERSITY OF SOUTHERN CALIFORNIA

CALIFORNIA INSTITUTE OF TECHNOLOGY

UNIVERSITY OF CALIFORNIA

MONTANA STATE UNIVERSITY

STANFORD UNIVERSITY

UNIVERSITY OF TOKYO

UNIVERSITY OF NEVADA

UNIVERSITY OF UTAH

NEW MEXICO STATE UNIVERSITY

WASHINGTON STATE UNIVERSITY

OREGON STATE UNIVERSITY

UNIVERSITY OF OREGON

OSAKA UNIVERSITY

UNIVERSITY OF WASHINGTON

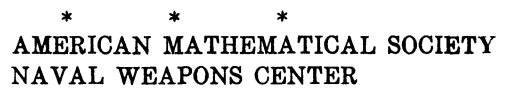

* C. DePrima will replace J. Dugundji until August 1974. 


\section{Pacific Journal of Mathematics}

\section{Vol. 47, No. $1 \quad$ January, 1973}

K. Adachi, Masuo Suzuki and M. Yoshida, Continuation of holomorphic

mappings, with values in a complex Lie group ....................

Michael Aschbacher, A characterization of the unitary and symplectic groups

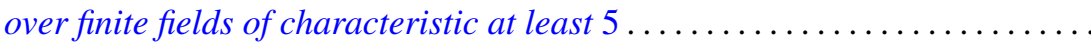

Larry Eugene Bobisud and James Calvert, Energy bounds and virial theorems for abstract wave equations....................................

Christer Borell, A note on an inequality for rearrangements ................

Peter Southcott Bullen and S. N. Mukhopadhyay, Peano derivatives and general

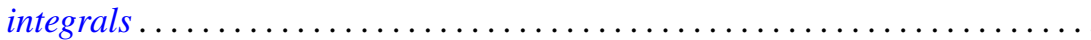

Wendell Dan Curtis, Yu-Lee Lee and Forrest Miller, A class of infinite dimensional subgroups of $\operatorname{Diff}^{r}(X)$ which are Banach Lie groups .........

Paul C. Eklof, The structure of ultraproducts of abelian groups ...............

William Alan Feldman, Axioms of countability and the algebra $C(X) \ldots \ldots \ldots$

Jack Tilden Goodykoontz, Jr., Aposyndetic properties of hyperspaces...........

George Grätzer and J. Płonka, On the number of polynomials of an idempotent algebra. II ...........................................

Alan Trinler Huckleberry, The weak envelope of holomorphy for algebras of

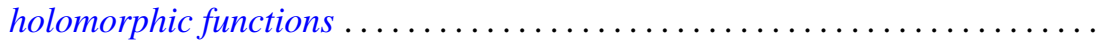

John Joseph Hutchinson and Julius Martin Zelmanowitz, Subdirect sum decompositions of endomorphism rings . . . . . . . . . . . . . . . .

Gary Douglas Jones, An asymptotic property of solutions of

$y^{\prime \prime \prime}+p y^{\prime}+q y=0$.

Howard E. Lacey, On the classification of Lindenstrauss spaces .

Charles Dwight Lahr, Approximate identities for convolution measure algebras.

George William Luna, Subdifferentials of convex functions on Banach

spaces.

Nelson Groh Markley, Locally circular minimal sets. .

Robert Wilmer Miller, Endomorphism rings of finitely generated projective modules

Donald Steven Passman, On the semisimplicity of group rings of linear

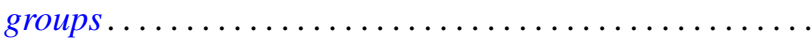

Bennie Jake Pearson, Dendritic compactifications of certain dendritic spaces.

Ryōtarō Satō, Abel-ergodic theorems for subsequences ...... .

Henry S. Sharp, Jr., Locally complete graphs. . .

Harris Samuel Shultz, A very weak topology for the Mikusinski field of

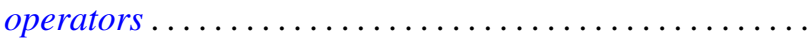

Elena Stroescu, Isometric dilations of contractions on Banach spaces ...

Charles W. Trigg, Versum sequences in the binary system ... . .

William L. Voxman, On the countable union of cellular decompositions of n-manifolds 